\title{
La subcontratación en la minería en Chile: elementos teóricos para el análisis*
}

\author{
Sandra Leiva Gómez ${ }^{* *}$
}

\begin{abstract}
Resumen: Las huelgas que han protagonizado en los últimos años los trabajadores subcontratados de la gran minería revelan que la ley de subcontratación no ha cumplido el objetivo de disminuir el conflicto entre trabajadores subcontratados, empresas subcontratistas y grandes empresas mineras. Este artículo analiza la subcontratación en la minería en Chile, entregando antecedentes teóricos y empíricos que permitan explicar tales conflictos. Desde un punto de vista sociológico, se alude a la pérdida de centralidad del trabajo asalariado y consiguiente aumento de formas de empleo atípicas. La empiria indica por otro lado un alto porcentaje de trabajadores en régimen de subcontratación en la minería. Se concluye que en algunas situaciones se presenta un encubrimiento laboral, que llevaría a la creación de trabajadores de primera y segunda categoría. Los trabajadores subcontratados afirman que deberían tener los mismos derechos y privilegios que sus pares contratados, porque realizan exactamente el mismo tipo de trabajo.
\end{abstract}

Palabras clave: subcontratación, industria minera, empleo precario, empleo atípico.

\section{Subcontracting in chilean mining: theoretical elements for analysis}

\begin{abstract}
Strikes carried out in recent years by subcontracted workers of the great mining reveal that the out-sourcing law has not fulfilled the objective of decreasing the conflict between subcontracted workers, subcontractors and large mining companies. This article analyzes the outsourcing in the mining in Chile, giving theoretical and empirical antecedents that allow explaining such conflicts. From a sociological point of view, refers to the loss of centrality of wage labor and subsequent growth of atypical employment. The available information points out on the other hand a high workers percentage in out-sourcing regime in the mining. It is concluded that in some situations is presented a disguised employment relationships, which lead to the creation of workers first and second class. Subcontracted workers assert that they should have same rights and privileges that his contracted pairs, because they carry out exactly the same type of work.

Key words: subcontracting, mining industry, precarious employment, atypical employment.
\end{abstract}

\footnotetext{
* El presente artículo fue presentado al Encuentro Pre-Alas Chile 2008, del 20 al 22 de Noviembre en Concepción, Chile.

** Universidad Arturo Prat, Departamento de Ciencias Sociales, Av. Arturo Prat 2120, Iquique, Chile, sandleiva@gmail.com
} 


\section{A subcontratação na minería em Chile: elementos teóricos para a análise}

Resumo: As greves que protagonizaram nos últimos anos os trabalhadores subcontratados da grande minería revelam que a lei de subcontratación não cumpriu o objetivo de diminuir o conflito entre trabalhadores subcontratados, empresas subcontratistas e grandes empresas mineiras. Este artigo analisa a subcontratação na minería em Chile, entregando antecedentes teóricos e empíricos que permitam explicar tais conflitos. Desde um ponto de vista sociológico, alude-se à perda de centralidade do trabalho assalariado e consiguiente aumento de formas de emprego atípicas. A empiria indica por outro lado uma alta percentagem de trabalhadores em regime de subcontratação na minería. Conclui-se que em algumas situações se apresenta um encubrimiento trabalhista, que levaria à criação de trabalhadores de primeira e segunda categoria. Os trabalhadores subcontratados afirmam que deveriam ter os mesmos direitos e privilégios que seus pares contratados, porque realizam exatamente o mesmo tipo de trabalho.

Palavras-chave: subcontratação, indústria mineira, emprego precário, emprego atípico.

Recibido: 26.08.2009

Aceptado: 26.10.2009

$* * *$

\section{Introducción}

Los conflictos de los trabajadores subcontratados del área de la minería han estado estos últimos años en el tapete del acontecer noticioso nacional, lo que da cuenta de la gran relevancia de este tema. Además, debido a que la legislación sobre esta materia es relativamente nueva -en enero de 2007 entró en vigencia la ley de subcontratación- se presentan conflictos que desde el punto de vista legal constituyen un desafío no sólo para abogados y jueces, sino también para el gobierno chileno. Por otra parte, estos conflictos revelan que hay aún variadas situaciones por resolver en este tema. De qué manera estos conflictos, enmarcados ahora en una regulación legal, pueden ser comprendidos y explicados desde la sociología en relación a la falta de protección que involucran, constituye el eje central de esta investigación.

El paradigma económico y sociopolítico neoliberal existente en Chile ha posibilitado la existencia de relaciones laborales altamente flexibilizadas, lo que ha implicado en variados casos un deterioro de las condiciones laborales y sociales de los trabajadores. La vuelta a la democracia del país no ha ido aparejada, sin embargo, de un restablecimiento de la protección de los trabajadores. Por otra parte, la subcontratación se entiende en el marco de una economía que ha debido adaptarse a condiciones cambiantes, llevando a las empresas a crear estrategias que flexibilicen el mercado laboral. De esta forma, las empresas han buscado la manera de abaratar los costos de su producción a través de mecanismos como la externalización, outsourcing y subcontratación, entre otros. Estos cambios han provocado 
profundas transformaciones en la sociedad, una de las cuales se refiere a la disminución del empleo clásico, estándar o normal. El trabajo asalariado ha ido perdiendo preeminencia, y frente a él, han aparecido modalidades atípicas de empleo. Estas nuevas formas de empleo implican en algunos casos una pérdida de protección, lo que se ha conceptualizado como empleo precario.

El presente artículo busca precisamente estudiar la subcontratación como ejemplo de una modalidad de empleo con insuficiente protección. Busca, además, explicar los conflictos de los trabajadores subcontratados en las grandes mineras a la luz de categorías analíticas aplicables a los empleos precarios o sin suficiente protección. Se postula que los conflictos de los trabajadores subcontratistas en la minería se deben a desigualdades de protección laboral y social y a desigualdades en las remuneraciones percibidas entre los trabajadores subcontratistas y sus pares de planta.

En la primera parte de este artículo se expone brevemente el problema de los trabajadores subcontratados en el área de la minería. En seguida se sitúa la subcontratación en el marco de la flexibilización del mercado laboral en Chile, en el contexto de un cambio de paradigma sociopolítico. Posteriormente se exponen elementos teóricos que permiten comprender de manera analítica la subcontratación. Tales elementos se refieren fundamentalmente a la aparición de nuevas formas de empleo y a la consiguiente disminución del empleo clásico o estándar, lo que da origen a situaciones altamente problemáticas para los trabajadores y para la sociedad en general. Luego se dan algunos antecedentes sobre la subcontratación en Chile desde sus orígenes hasta el presente, aportando información acerca de los sectores de la economía que más subcontratan y de su evolución en el tiempo. En el siguiente apartado se entregan datos concretos de la subcontratación en el sector minero, mostrando cifras sobre las empresas mandantes y contratistas, y sobre el número de trabajadores contratados en unas y otras. Por último, este texto finaliza con un análisis de la subcontratación basado en los elementos teóricos entregados anteriormente.

\section{Los conflictos de los trabajadores subcontratados en la minería}

En los últimos años los trabajadores subcontratados por las empresas mineras han protagonizado serios conflictos, aun cuando ya existe una regulación de la subcontratación. Diversas divisiones de la Corporación Nacional del Cobre -CODELCO- han recurrido a la justicia, apelando en contra de las disposiciones dictadas por la Dirección del Trabajo, referida a la internalización de trabajadores subcontratados. El fundamento legal de ello es el artículo 183 A del Código del Trabajo -disposiciones introducidas por la ley de subcontratación- en que se considera a determinadas empresas mineras como empleadores de estos trabajadores. Las cortes de apelaciones de Valparaíso y Antofagasta fallaron en el año 2007 a favor de las 
empresas mineras, en tanto que la Corte de Copiapó rechazó el recurso que la minera de El Salvador había presentado. El 12 de mayo fallaron las Cortes de Apelaciones a favor de CODELCO y de la Minera La Escondida. Aún cuando se reconoce la falta a la ley de las mineras, no se reconoce a la Dirección del Trabajo la capacidad de hacer cumplir la ley a través de dar una orden de contratación de los trabajadores.

Uno de los grandes conflictos laborales vivenciados por CODELCO durante el año 2007 tiene su origen en la mejora de salarios (Daroch 2007). Este conflicto se caracterizó por una masividad y amplia convocatoria, articulándose desde los mismos trabajadores, en segundo lugar, un liderazgo joven en relación a otros conflictos laborales, siendo su motivación el superar antiguas prácticas sindicales, en tercer lugar el conflicto responde a lógicas del movimiento y no a partidos políticos, en cuarto lugar la violencia en las manifestaciones y en quinto lugar una gran cobertura en los medios de comunicación (Daroch 2007). En este conflicto los trabajadores decidieron que el interlocutor para la negociación no sería el empleador directo -la empresa subcontratista- sino CODELCO (Daroch 2007).

La huelga en CODELCO se produjo por las condiciones desiguales de salario y de ausencia de ciertas garantías de los trabajadores subcontratados en relación a sus pares contratados (Daroch 2007). El primer paro de los trabajadores subcontratados comienza a fines de 2005 e inicios de 2006. Posteriormente comienzan negociaciones entre las empresas contratistas, CODELCO y los trabajadores subcontratados, originándose dentro de este marco en junio de 2007 una de las manifestaciones más violentas y masivas de los últimos años. Tal manifestación concluye el 31 de julio, luego de 36 días de huelga. Se llegó a acuerdo al firmar entre los trabajadores y CODELCO un bono de aproximadamente \$ 450.000 y el pago de 8 días de huelga, además de ciertos beneficios en salud y seguros de vida (Daroch 2007).

\section{La flexibilización del mercado laboral en Chile}

Para entender la subcontratación en Chile debe hacerse mención a las transformaciones económicas en América Latina en los últimos 30 años y las circunstancias en que éstas se llevaron a cabo. Debido a la incapacidad de América Latina de cancelar la deuda externa en la década de los ochenta, se implementaron una serie de reformas económicas que dieron por resultado un cambio de paradigma económico, político y sociopolítico, entre otros. Los países latinoamericanos, que hasta entonces habían seguido un modelo de desarrollo "hacia adentro" impulsado por la CEPAL bajo la modalidad de la industrialización por sustitución de importaciones, debieron cambiar su estilo de desarrollo. Cabe destacar que este cambio de estrategia no fue calculado, sino más bien fue la resultante de imposiciones que las organizaciones financieras internacionales -tales como el Fondo Monetario Internacional y el Banco Mundial- le impusieron a la región para continuar recibiendo créditos. De esta forma empezó a implementarse 
paulatinamente en los diversos países de América Latina el neoliberalismo económico, basado en las ideas de Milton Friedman sobre la economía del lado de la oferta.

En Chile las reformas laborales comenzaron a implementarse ya en la década del setenta luego de que asumiera el gobierno militar. En el año 1979 se promulgaron tres importantes leyes -el plan laboral-, que tendían a flexibilizar fuertemente el mercado laboral. A través de la promulgación de éstas y otras leyes que le siguieron, las relaciones laborales ${ }^{1}$ tanto individuales como colectivas se tornaron altamente flexibilizadas. Con la vuelta del país a la democracia se esperaba que el gobierno realizara cambios significativos en material laboral, entre otros. Una aspiración consistía en restituir los derechos laborales individuales y colectivos que se habían ido perdiendo producto de las medidas flexibilizadoras. Aun cuando durante los gobiernos de la concertación se han ido realizando sucesivas reformas al Código del Trabajo, permanecen importantes normas que dan cuenta de la todavía inmensa flexibilidad laboral. Ejemplo de esto es el artículo 161 del Código del Trabajo, que permite el despido de trabajadores aludiendo a necesidades de la empresa, sin que medien criterios o especificaciones para tales necesidades. En materia de relaciones laborales colectivas, los niveles de sindicalismo que ya habían disminuido drásticamente debido a la dictadura, no han logrado recuperarse, e incluso han continuado disminuyendo en democracia.

\section{Elementos teóricos para el análisis de la subcontratación: nuevas formas de empleo}

En Chile los estudios de la desprotección de los trabajadores han sido realizados en su mayoría por investigadores ligados a la Dirección del Trabajo (Henríquez y Echevarría 2006, Echeverría 2006, Ugarte 2008). El problema de la subcontratación ha sido investigado en nuestro país desde el punto de vista jurídico (Ugarte 1999, Albornoz 2001, Ugarte 2007). El concepto jurídico que marca la frontera entre los trabajadores asalariados y los independientes es el de la dependencia (Ugarte 1999, Feres, Henríquez y Ugarte 1999). En efecto, una relación laboral se constituye como tal en cuanto el trabajador tiene una relación de dependencia o subordinación con su empleador (Albornoz 2001). Si esta dependencia o subordinación está ausente, entonces la relación no constituye una relación laboral, sino civil o comercial. La persona ocupada no está protegida por las leyes del código laboral, por cuanto ésta no tiene la calidad jurídica de trabajador. El gran problema es, por tanto, determinar en qué casos se da esta relación de

\footnotetext{
${ }^{1}$ Se distinguen en Chile tres etapas en materia de relaciones laborales. La primera, que va desde 1931 a 1973, está marcada por un proteccionismo laboral, la segunda, de 1974 a 1989, ha estado orientada más a los empresarios, mientras que la tercera etapa, de 1990 hasta nuestros días, es más proclive a los trabajadores que la anterior, aunque no se vuelve a los niveles de protección laboral del primer período (Thiery 2000, Valencia 1998).
} 
dependencia entre el trabajador y el empleador y en cuáles no. Aun cuando éste es un problema exclusivamente jurídico, pues tal determinación corresponde a las instancias que tienen la labor de hacer cumplir la ley laboral, desde un punto de vista sociológico es posible encontrar luces que permitan explicar los conflictos en materia de subcontratación.

A pesar de que ahora existe la Ley de Subcontratación ${ }^{2}$ en nuestro país, los problemas entre trabajadores y empresas no se han solucionado. A fin de comprender y explicar el descontento de los trabajadores subcontratistas específicamente de la minería, se opta aquí por una teoría de alcance medio: el enfoque del empleo clásico o normal, llamado también trabajo asalariado. Este enfoque constituye un marco teórico que permite comprender la subcontratación de manera analítica, y con ello, explicar, de una manera sociológica, los conflictos laborales que han sido vividos en los últimos años en la gran minería en Chile. A continuación se expondrán entonces los conceptos pertinentes para el análisis de la subcontratación, contextualizados en los cambios que han sufrido las empresas en el mundo entero.

La economía mundial ha experimentado grandes cambios en las últimas décadas. A fin de adaptarse a las condiciones cambiantes de la economía, las empresas han debido desarrollar una serie de estrategias orientadas a mantener su competitividad, dando así origen a la flexibilidad del mercado laboral. De esta manera, las empresas han buscado formas alternativas de trabajo, distintas al trabajo asalariado. Los costos no laborales asociados a la productividad -pago de cotizaciones previsionales, pago de feriados, vacaciones, maternidad, entre otros- les resultan demasiado altos a las empresas. A fin de reducir estos costos, éstas han ideado estrategias para ahorrárselos. Una de esas estrategias consiste en sustituir al trabajador por personas que prestan servicios, dando origen así a la externalización, tercerización, outsourcing y subcontratación. Las empresas hoy en día se valen de estas estrategias en todo el mundo -y nuestro país no está ajeno a ellas- para disminuir sus costos y poder competir con otras de su rubro.

Tales estrategias desarrolladas por las empresas van originando nuevas formas de empleo, lo que trae por consecuencia una disminución del trabajo asalariado. Así, porcentualmente las nuevas formas de empleo aumentan, mientras que el trabajo asalariado decae. Un serio problema es que muchas veces estas nuevas formas de empleo son precarias, es decir, no ofrecen una seguridad al trabajador, ni en términos de previsión social, ni en términos de condiciones laborales. Otras veces presentan incluso remuneraciones inferiores. Estas nuevas formas de empleo han sido conceptualizadas en la literatura como empleos atípicos, puesto que se apartan en una o más características de un empleo asalariado que ofrece normas básicas y estándares (Rodgers 1988). Al desviarse los empleos atípicos de las normas estándares, en la mayoría de las ocasiones devienen en una precariedad.

${ }^{2}$ La Ley de Subcontratación será analizada en los siguientes apartados. 
El constructo teórico de empleo normal (Leiva 2006) permite comprender los llamados empleos atípicos, los que se definen por oposición a la norma establecida, vale decir, los empleos atípicos son aquellos que se salen de la norma, desviándose en una u otra característica, o en varias de ellas, del empleo de carácter normal. En este punto es necesario referirse a los empleos precarios, que también se salen de esta norma. Es importante dejar en claro que empleos atípicos y empleos precarios no son expresiones sinónimas, aun cuando hasta hace pocos años todavía eran considerados sinónimos (OIT 1998). Los empleos precarios son aquéllos que adolecen de protección en algún ámbito importante (Rodgers 1988). Una diferencia fundamental entre empleos atípicos y empleos precarios es que los empleos atípicos no necesariamente carecen de protección (Leiva 2000, Leiva 2006), lo que puede apreciarse más claramente al observar los tipos de empleo existente en otros países, ${ }^{3}$ como es el caso de Alemania. ${ }^{4}$ En ese país, muchos de los empleos atípicos fueron reglamentados a fin de que se les otorgara protección en diversos ámbitos. De esta manera, esos empleos son atípicos, pero no precarios.

La distinción teórica entre empleos atípicos y empleos precarios es clave para comprender el fenómeno de la subcontratación en Chile, porque constituye una forma de empleo atípica, y porque esta forma de empleo atípica es además precaria al no contar con la protección necesaria. Precisamente esta ausencia de protección es uno de los talones de Aquiles de esta forma de empleo, y lo que podría explicar los conflictos y huelgas de trabajadores que se han suscitado en las empresas subcontratistas de la gran minería.

\section{La subcontratación en Chile}

\section{a) Aspectos legales}

La figura del subcontratista surge en Chile el año 1975 con la dictación de la Ley número 1.263, que establece labores de subcontratación con ciertas restricciones. Así, se excluían del régimen de subcontratación las labores inherentes a la producción principal y permanente de la empresa. Además, la subcontratación se limitaba a la reparación o mantención de equipos. El Decreto ley número 16.757 de 1979 amplía las funciones de los subcontratistas que habían sido establecidos por la ley de 1975, haciendo posible subcontratar trabajadores para todas las áreas de las empresas (Silva 2007). En los años ochenta y noventa varias trasnacionales aumentaron todavía más la figura de la subcontratación. Con la llegada al país de em-

\footnotetext{
${ }^{3}$ Para conocer varias formas atípicas de empleo en países europeos occidentales y estudiar la protección que se les otorga, ver Emmerich 1999.

${ }^{4}$ Ejemplos de formas de empleo atípicas en Alemania que poseen protección legal y social son el trabajo a tiempo parcial (Teilzeitarbeit), el trabajo suministrado (Leiharbeit) y las ocupaciones por una mínima cantidad de horas (geringfügige Beschäftigungsformen).
} 
presas de servicios temporarios, tales como Manpower, Ecco y Part-Time en la década de los 80, la subcontratación se generalizó aún más (Silva 2007). Por una parte existía la práctica de la subcontratación, que cada vez se fue masificando más, y por otra parte existía el trabajo suministrado, figuras que tendieron a confundirse. Paulatinamente se fueron presentando conflictos y situaciones irregulares, y al no haber una ley que regulara ni el trabajo en régimen de subcontratación ni el trabajo suministrado, se hacía difícil su tratamiento legal. Si bien es cierto la economía se benefició de estos cambios, esto trajo consigo una disminución y en ocasiones una pérdida de la protección que el derecho laboral y social le otorgaban a los trabajadores.

La ley de subcontratación, aprobada en el año 2006, y que empezó a regir en enero de 2007, ${ }^{5}$ reconoce dos figuras distintas: por una parte la subcontratación laboral propiamente tal, y por otra, el suministro de trabajadores $^{6}$ (Ugarte 2006, Dirección del Trabajo 2007). Tanto en la figura de la subcontratación -definida en términos de esta ley- como en la figura del suministro de trabajadores se distinguen tres actores claves: en primer lugar la empresa mandante o bien la empresa usuaria, en segundo lugar la empresa contratista o bien la empresa suministradora y en tercer lugar los trabajadores. Ambas figuras se distinguen por las relaciones -laborales o comerciales- y por las implicancias jurídicas que se derivan, tanto para las empresas como para los trabajadores, de tales relaciones.

La subcontratación se refiere a la situación en que una empresa mandante o principal le encarga a otra, la empresa contratista, la producción de bienes o la prestación de servicios. La empresa contratista desarrolla la labor de forma autónoma, llevándola a cabo por su cuenta y con sus propios recursos monetarios, materiales y humanos. En este caso se establece una relación laboral entre la empresa contratista y los trabajadores que tiene a su cargo, mientras que entre la empresa mandante y la empresa contratista se establece una relación comercial de prestación de servicios. Cabe hacer notar que los trabajadores y la empresa mandante no establecen una relación laboral, es decir, los trabajadores desarrollan sus tareas para la empresa contratista.

Por su parte, la figura de suministro de personal corresponde a una empresa usuaria y una empresa que presta sus trabajadores, llamada empresa suministradora. En este caso, a diferencia del anterior, los trabajadores establecen una relación laboral con la empresa usuaria, puesto que reciben instrucciones directamente de ésta, y laboran bajo su dependencia. A pesar de que el trabajador establece un vínculo labo-

\footnotetext{
${ }^{5}$ La ley 20123 fue publicada el día 16 de Octubre de 2006 y entró en vigencia el 14 de Enero de 2007.

${ }^{6}$ Cabe destacar que estas denominaciones se prestan para confusión, porque la ley ha precisado el concepto de subcontratación de manera restringida, aun cuando el concepto se ocupa en la literatura de manera amplia. A juicio de la investigadora la denominación de régimen de subcontratación otorgado al primer tipo de empresa es poco feliz por cuanto contribuye a confundir (aún más que antes) a los trabajadores y a la opinión pública.
} 
ral con la empresa usuaria, es la empresa suministradora quien le paga su salario, lo que lleva a afirmar que la figura del empleador aparece en esta figura disociada.

Ha de notarse que la diferencia fundamental entre una y otra figura es la dependencia de los trabajadores: mientras que en el caso de la subcontratación la dependencia de los trabajadores se establece con respecto a la empresa contratista, en el caso del suministro de trabajadores la dependencia de los trabajadores queda establecida con respecto a la empresa usuaria. Esta dependencia es de vital importancia pues determina el tipo de contrato que se establece entre los trabajadores y las empresas. Cuando existe una relación laboral, corresponde firmar un contrato indefinido o en su defecto un contrato a plazo fijo, los que están sujetos a las normas del código laboral. Cuando no existe una relación laboral, sino civil o comercial, corresponde firmar un contrato a honorarios, el que se rige por los códigos civil y comercial. Mientras los primeros son considerados trabajadores y gozan de todos los beneficios laborales y previsionales que las leyes les reconocen, los segundos no son considerados trabajadores y por lo tanto no están sujetos a las normativas del código laboral.

Es necesario reconocer en esta dicotomía de contratos laborales y civiles un problema de gran relevancia y aún sin resolver. En un mundo globalizado, en constante cambio, cuyos cambios ocurren muchas veces a velocidades vertiginosas, las empresas se ven obligadas a reaccionar con suficiente celeridad ante una demanda por bienes y servicios que oscila en el tiempo, optando por eludir la contratación indefinida e incluso en ocasiones la contratación a plazo fijo. De esta forma, numerosas empresas prefieren utilizar contratos más flexibles, para en un momento de baja demanda por sus bienes o servicios, poder prescindir de su personal sin tener que incurrir en gastos indemnizatorios.

\section{b) La subcontratación en cifras}

Existe una dificultad para comprender y estudiar la subcontratación a nivel nacional debido a que los instrumentos de carácter nacional actuales no dan cuenta de los trabajadores subcontratados, ${ }^{7}$ sea que trabajen en régimen de subcontratación en sentido estricto -sentido de la ley de subcontratación- sea que trabajen en empresas usuarias. En otros países, las empresas deben registrarse en la inspección de trabajo que según su jurisdicción debe controlar laboralmente. En Chile no existe un registro de este tipo para efectos de control de la legislación laboral, lo que hace difícil la recopilación estadística (Silva 2007). Además, tampoco existe en nuestro país un registro de empresas contratistas y de empresas suministradoras de personal, ${ }^{8}$ tampoco de la cantidad de personas que trabajan bajo un

\footnotetext{
${ }^{7}$ Ni la Encuesta de Empleo del Instituto Nacional de Estadísticas, ni la Encuesta CASEN, ni el Servicio de Impuestos Internos registran la subcontratación.

${ }^{8}$ Solamente en el sector de la minería existe un registro de este tipo: El Servicio Nacional de Geología y Minería (SERNAGEOMIN) ha dispuesto que las mineras lleven un registro de la
} 
régimen de subcontratación (Silva 2007). Es por ello que en el estudio de la subcontratación se utiliza la Encuesta Laboral de la Dirección del Trabajo (ENCLA) ${ }^{9}$ como referencia, la cual busca completar la información proporcionada por encuestas nacionales en relación al fenómeno de la subcontratación. ${ }^{10}$ Aun cuando la ENCLA no tiene un carácter de aplicación nacional, es el instrumento que está en mejores condiciones para medir la subcontratación a nivel nacional. ${ }^{11}$

Según la ENCLA 2006, las empresas ${ }^{12}$ que subcontratan alcanzan el $41,2 \%$. Cabe destacar que las empresas que más subcontratan son las de mayor tamaño. De acuerdo a la ENCLA 2006 la gran empresa subcontrata a un $68,3 \%$, la mediana empresa $58,7 \%$, la pequeña empresa $40,7 \%$ y la microempresa $^{13}$ 27,9\% (Dirección del Trabajo 2007), lo que hace a la gran empresa especialmente atractiva en el estudio de la subcontratación. Por otra parte, las ramas que porcentualmente más subcontratan según la ENCLA 2006 son en primer lugar las empresas de electricidad, gas y agua, en segundo lugar los sectores de servicios sociales y de salud, en tercer lugar construcción, en cuarto lugar el sector industrias, y en quinto lugar la minería (Dirección del Trabajo 2007). Cabe destacar que en al año anterior de aplicación de la Encuesta, la ENCLA 2004, registraba a la minería como el segundo sector con más participación en subcontratación.

Otro dato importante a considerar son los sectores en donde la subcontratación ha aumentado más. En este sentido, la minería es el sector donde más ha aumentado porcentualmente la subcontratación: de un 37\% en el año 1999 ascendió a un 63,8\% en el año 2004, registrándose un aumento de 26 puntos porcentuales, lo que resulta considerable de tener en cuenta.

cantidad de empresas subcontratistas y de los trabajadores que laboran en empresas mandantes y contratistas (Silva 2007).

${ }^{9}$ La encuesta laboral realizada por la Dirección del Trabajo, ENCLA, reconoce que hay varios aspectos en relación al trabajo que no se pueden medir con los instrumentos en el país hasta ahora existentes, entre ellos, la subcontratación. Por ello se implementó la ENCLA en el año 1998, y se ha aplicado posteriormente en los años 1999, 2002, 2004 y 2006.

${ }^{10}$ Aun cuando en el año 2006 la Encuesta CASEN considera los trabajadores suministrados al introducir una alternativa de respuesta referida a los servicios transitorios, este instrumento continúa resultando insuficiente para registrar la subcontratación.

${ }^{11}$ La última Encuesta ENCLA, llevada a cabo el año 2006, considera diez regiones de aplicación, entre las que quedan excluidas -por una razón de costo (Dirección del Trabajo 2007)la antigua región de Tarapacá, la región de Aysén y la región de Magallanes. Sin embargo, en Aysén se dispone de una encuesta similar del año 2003, en tanto que en Magallanes existe un proyecto a llevar a cabo (ENCLA 2006).

${ }^{12}$ La ENCLA considera aaproximadamente el 2\% del marco muestral de empresas. Se entrevistaron a 1.281 empresas en las diez regiones señaladas. El 2\% corresponde a 1560 empresas, con un nivel de logro del 82\%, lo que da por resultado un total de 1.281 empresas encuestadas (ENCLA 2006, Dirección del Trabajo 2007).

${ }^{13}$ Se considera gran empresa a las que poseen 200 trabajadores o más, mediana empresa de 50 a 199 trabajadores, pequeña empresa de 10 a 49 y microempresa de 5 a 9 trabajadores. 


\section{La subcontratación en la minería}

Según se vio en el apartado anterior, la minería es una de las ramas de actividad económica que más utiliza la figura de la subcontratación (Dirección del Trabajo 2007, Echeverría 2006). Como se había mencionado anteriormente, la minería es la única rama de actividad económica que lleva, por disposición del Servicio Nacional de Geología y Minería (SERNAGEOMIN), un registro de la subcontratación. En él se contemplan las empresas mandantes, las contratistas, el personal propio y el personal subcontratado (Echeverría 2006). A continuación se revisarán tales datos para ver la cantidad de empresas mineras - detallando entre empresas contratistas y mandantes- y su evolución en el tiempo. Además, se revisará la cantidad de trabajadores que se ocupan en estas empresas, realizando también un análisis de su tendencia en el tiempo.

En primer lugar, debe notarse un considerable aumento en el sector de la minería. Este aumento se refleja tanto en la cantidad de empresas mineras que existen (ver gráfico número 1) como en la cantidad de trabajadores empleados en este rubro (ver gráfico número 3). En el sector minero se distinguen empresas mandantes y empresas contratistas, siendo esta distinción la que precisamente motiva este artículo. Las empresas mandantes le encargan a las empresas contratistas la ejecución del trabajo, el que debe realizar bajo su propia cuenta y riesgo. La empresa contratista aporta sus propios trabajadores para este fin. De esta forma, existen trabajadores que están contratados por las empresas mandantes y trabajadores contratados por las empresas contratistas.

Al analizar los datos de las empresas mineras desde 1997 hasta 2006, se observa claramente un alza en el total de las empresas (ver tabla número 1). Debe repararse en que esta alza es constante, vale decir, año tras año las empresas mineras han aumentado. Además, se trata de un aumento no sólo constante, sino considerable, puesto que en diez años las empresas mineras han aumentado en un $270 \%$, es decir, casi se han triplicado. En efecto, el número de empresas mineras era de 1322 en 1997, y en el año 2006 llegó a 3628.

Tabla Número 1

Empresas mandantes y contratistas en la minería en Chile en el período 1997 a 2006

\begin{tabular}{|l|r|r|r|r|r|r|r|r|r|r|}
\hline Año & 1997 & 1998 & 1999 & 2000 & 2001 & 2002 & 2003 & 2004 & 2005 & 2006 \\
\hline Mandantes & 403 & 338 & 302 & 314 & 310 & 293 & 300 & 341 & 360 & 471 \\
\hline Contratistas & 919 & 1031 & 1079 & 1296 & 1358 & 1501 & 1839 & 2033 & 2269 & 3157 \\
\hline Total & 1322 & 1369 & 1381 & 1610 & 1668 & 1794 & 2139 & 2374 & 2629 & 3628 \\
\hline
\end{tabular}

Fuente: Elaboración propia, en base a datos de SERNAGEOMIN. 
Al observar más detalladamente, según sean éstas empresas mandantes o contratistas, se aprecia una notoria diferencia entre la evolución de ambos tipos de empresas. Mientras que las empresas contratistas han aumentado en forma constante, la evolución de las empresas mandantes muestra más bien una desarrollo numéricamente constante. Ambas tendencias pueden observarse más claramente al observar los datos en términos porcentuales (ver gráfico número 1). Las empresas contratistas representaban en 1997 el 69,5\% del total de empresas mineras. Este porcentaje ha ido aumentando constantemente, de tal manera que diez años después las empresas contratistas representan el 87\% del total, observándose un aumento de más de 17 puntos porcentuales. Concordantemente, las empresas mandantes han disminuido su participación porcentual en las empresas mineras. En el año 1997 las empresas mandantes representaban casi la tercera parte de las empresas mineras (30,5\%), llegando en el año 2006 a sólo un 13\%. En el análisis cabe destacar que las empresas mandantes han reducido su participación porcentual dentro del total de empresas mineras del país en más de la mitad tan sólo en diez años.

\section{Gráfico Número 1}

Empresas mandantes y contratistas en la minería en Chil en el período 1997 a 2006 en porcentajes

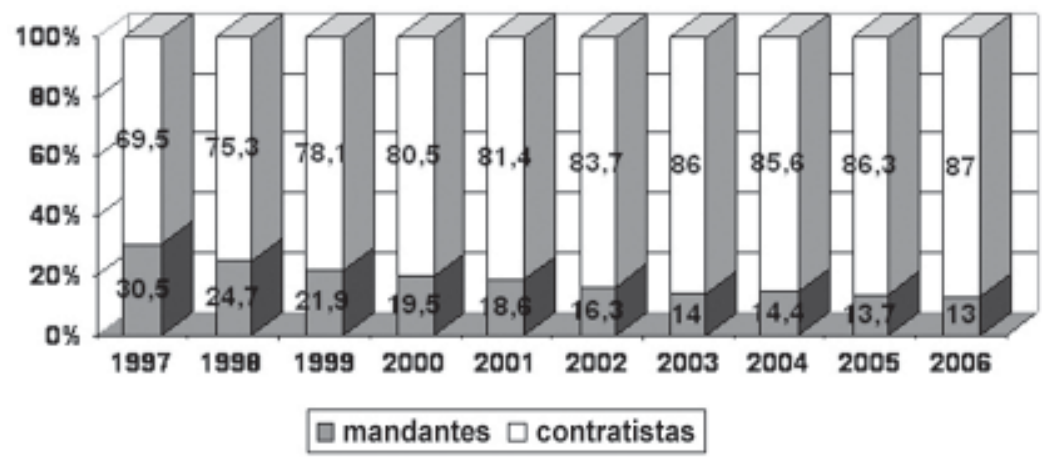

Fuente: Elaboración propia en base a datos de SERNAGEOMIN.

Por otra parte, la cantidad de trabajadores ocupados en la minería ha crecido ostensiblemente. Mientras en el año 1985 existían 67.100 trabajadores empleados en este rubro, en el año 2005 hay 133.989 trabajadores. En otras palabras, en 20 años prácticamente se duplicó la cantidad de trabajadores empleados en el sector minero (ver tabla número 2). 
Tabla Número 2

Número de trabajadores de empresas mandantes y contratistas en la minería en el período 1985 a 2006

\begin{tabular}{|l|r|r|r|r|r|}
\hline Año & 1985 & 1990 & 1995 & 2000 & 2005 \\
\hline Contratistas & 3174 & 10751 & 27300 & 39476 & 85891 \\
\hline Mandantes & 63926 & 74508 & 54938 & 46621 & 48098 \\
\hline Total & 67100 & 85259 & 82238 & 86097 & 133989 \\
\hline
\end{tabular}

Fuente: Elaboración propia, en base a datos de SERNAGEOMIN.

La cantidad de trabajadores en las empresas contratistas y en las empresas mandantes es, del mismo modo que la cantidad de las empresas contratistas y mandantes, sorprendente. En el año 1985 existía un bajo porcentaje $(4,7 \%)$ de trabajadores empleados en empresas contratistas, en el año 2005 este porcentaje crece en más de trece veces, llegando a haber $64,1 \%$ de trabajadores ocupados en empresas contratistas (ver gráfico número 2). Mientras que los trabajadores mineros que se emplean en empresas contratistas aumentan drásticamente, los trabajadores de la minería ocupados en empresas mandantes disminuyen igualmente de un modo drástico su participación porcentual.

\section{Gráfico Número 2}

Número de trabajadores de empresas mandantes y contratistas en la minería en Chile en porcentajes en el período 1985 a 2006

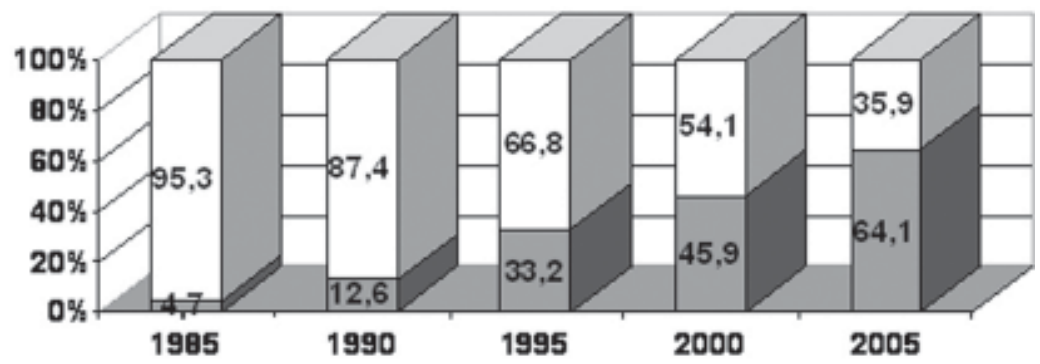

\section{número de trabajadores en empresas mandantes \\ número de trabajadores en empresas contratistas}

Fuente: Elaboración propia, en base a datos de SERNAGEOMIN. 
En conclusión, el sector minero ha crecido ostensiblemente. Este crecimiento se aprecia tanto en cantidad de empresas mineras como en la cantidad de trabajadores empleados en este rubro. Debe notarse que este crecimiento es significativo en las empresas contratistas, puesto que las empresas mandantes han disminuido porcentualmente. Además, debe tenerse presente que cada vez hay una participación más importante de los trabajadores en las empresas contratistas, con la consiguiente significativa disminución porcentual de trabajadores en empresas mandantes. Cada vez hay más empresas contratistas. Cada vez hay más y más trabajadores en estas empresas. No es de extrañar, entonces, que al presentarse conflictos laborales en las empresas contratistas, haya un gran número de trabajadores involucrados en tales conflictos.

\section{Análisis de la subcontratación a la luz de los elementos teóricos}

Se presentan variados aspectos problemáticos en relación a la ley de subcontratación. Uno de ellos se refiere a las diferencias en cuanto a derechos que se presentan entre los trabajadores subcontratados y sus pares contratados. Esto lleva a darse cuenta de que los tipos de protección que la ley ofrece a los trabajadores no es homogéneo, por cuanto algunas formas subcontratación tendrían grados menores de protección que los trabajadores de planta. Estas diferencias llevan a pensar en la existencia de trabajadores de primera y segunda categoría. Además, otra diferencia está dada por la desigualdad de remuneraciones entre los trabajadores de la empresa usuaria o de la empresa mandante (según sea el caso) y las remuneraciones de los trabajadores en empresas suministradas o en empresas subcontratistas respectivamente (Ugarte 2008).

Otro aspecto problemático en la ley de subcontratación se debe a la dificultad de determinar en ciertas ocasiones si una empresa es mandante $o$ usuaria. Vale decir, si una empresa que hace uso de trabajadores externos lo hace bajo la forma -hoy legal- de subcontratación o de suministro de personal, con independencia de la denominación que ésta le dé. Según la ley, existe subcontratación cuando no existe potestad de mando entre la empresa que utiliza los servicios y los trabajadores, y existe por el contrario trabajo suministrado cuando esta potestad de mando sí está presente entre ambos. Determinar cuándo existe esa potestad de mando y cuándo no, se torna en ocasiones altamente complejo. Precisamente éste ha sido uno de los focos de conflicto, entre otros, desde que la Dirección del Trabajo comenzó a fiscalizar a algunas empresas subcontratistas luego de que entrara en vigencia la ley de subcontratación.

Cabe destacar que la huelga de los trabajadores subcontratados en la minería fue ilegal. En este punto es importante destacar que de acuerdo al marco jurídico vigente no proceden las huelgas en las empresas subcontratistas. Las huelgas están reglamentadas en situaciones laborales, y al existir un régimen jurídico comercial o civil, no se considera un dere- 
cho a huelga. La legislación laboral otorga el derecho a una negociación colectiva en el caso de un contrato de trabajo indefinido o a plazo fijo. En el caso de un trabajador en régimen de subcontratación no procede una negociación colectiva. Así lo establece el código del trabajo en su artículo 305 número 1 "Los trabajadores (...) que se contraten exclusivamente para el desempeño en una determinada obra o faena transitoria o de temporada" no pueden negociar colectivamente. Aun cuando las huelgas en materia de subcontratación son ilegales, en la opinión pública existen voces de que el Gobierno debe tomar cartas en el asunto y regular este tipo de relaciones laborales a fin de evitar estos conflictos "Lo que ha ocurrido (...) es un llamado de alerta muy serio al Gobierno respecto de su regulación en materia laboral y a los dirigentes sindicales y a los empresarios, para buscar la sensatez y caminos de entendimiento sólidos.”14

Otro aspecto que debe ser considerado dice relación con la bonanza económica de las empresas en que participan los trabajadores subcontratados. Los trabajadores notan las abundantes ganancias económicas, y ellos quieren ser parte también de esta bonanza (Daroch 2007). Así, Felipe Morandé afirma que "esos problemas no surgen de la nada, sino que son fruto de la sensación de riqueza que existe en el país y de la cual gran parte de la ciudadanía se siente al margen." ${ }^{15}$ En el caso de CODELCO hay que agregar el alto precio del cobre (Daroch 2007), factor que debería tenerse presente a la hora de analizar las demandas de los trabajadores del cobre. Y a pesar de que los trabajadores contratados bajo un régimen de subcontratación no tienen derecho a una negociación colectiva -entre varios otros derechos más-, ello no les impide sentir legitimidad para clamar por mejoras salariales, especialmente cuando la empresa para la cual trabajan experimenta enormes ganancias económicas.

A pesar de sus avances, la ley de subcontratación presenta serios problemas, pues no da cuenta de situaciones problemáticas para los trabajadores subcontratados. Ellos se refieren principalmente a la diferencia en las condiciones de salario y en las condiciones laborales entre los trabajadores por una parte subcontratados y suministrados y por otra parte los trabajadores directos de la empresa principal (Silva 2007). Estas diferencias permiten hablar de una discriminación entre estos dos tipos de trabajadores, con la cual se estarían creando trabajadores de primera y de segunda categoría. Los trabajadores de primera categoría corresponderían a los trabajadores asalariados, es decir, se ocupan bajo la forma de un empleo normal o estándar. A ellos está dirigido el ordenamiento del Código del Trabajo, y se les garantiza la máxima protección que el ordenamiento jurídico puede garantizar. Los trabajadores subcontratados por las empresas mineras contratistas constituirían una especie de trabajadores de segunda cate-

\footnotetext{
${ }^{14}$ Cristián Saieh, Director del Centro de Negociación, Mediación y Arbitraje de la Universidad Católica, citado por Daroch 2007.

${ }^{15}$ Felipe Morandé, Decano de la Facultad de Economía y Negocios de la Universidad de Chile, en el Diario Financiero, 24.08.2007, citado por Daroch 2007.
} 
goría. El grado de protección ofrecido a los trabajadores subcontratados no es tan alto como en el caso de los trabajadores de primera categoría. Y puede existir incluso el peligro de que las empresas contratistas no asuman su responsabilidad en el pago de cotizaciones previsionales. Afortunadamente, esta última situación se reglamentó en la ley de subcontratación, sin embargo, la alternativa que se propone en la ley, que la empresa mandante asuma solidariamente la responsabilidad del pago de cotizaciones, no está exenta de problemas y situaciones engorrosas.

Otra área problemática que vivencian los trabajadores subcontratados se refiere a las remuneraciones ${ }^{16}$. Existe una gran diferencia entre las remuneraciones que perciben los trabajadores contratados por la empresa mandante y los trabajadores contratados por las empresas contratistas, a pesar de que ambos realizan el mismo tipo de trabajo. Esta situación es catalogada por los subcontratistas como injusta. Para lograr una compensación remuneratoria, en ocasiones los trabajadores subcontratados han decidido negociar no con la empresa de la cual dependen directamente, la empresa contratista, sino con la empresa mandante, pues es ésta quien se lleva las mayores ganancias y la que está en condiciones financieras de otorgarles un bono de remuneración que les satisfaga de acuerdo a sus percepciones de justicia.

Además, existe una seria dificultad en relación a la calificación de una empresa como contratista o como suministradora de servicios. El concepto clave que hace la diferencia es la subordinación o dependencia. Si el trabajador realiza sus labores bajo subordinación o dependencia de la empresa contratista, establece efectivamente un claro vínculo laboral entre esta empresa y los trabajadores. Por el contrario, si los trabajadores se ocupan bajo la subordinación y dependencia no de la empresa contratista, sino de la empresa mandante, entonces no se establece un vínculo laboral entre los trabajadores y la empresa contratista. El vínculo laboral se establece en este caso entre los trabajadores y la empresa mandante. Este caso es altamente conflictivo.

Al establecer el vínculo laboral entre la empresa mandante y los trabajadores subcontratados, caben dos posibilidades: se trata de trabajo suministrado, o bien se está encubriendo una situación laboral sacando provecho de esta situación en desmedro de los derechos de los trabajadores.

\footnotetext{
${ }^{16}$ Mención aparte merece la diferencia entre el mecanismo para la determinación de salarios en mercados no sindicalizados y sindicalizados. Según Mc Connel et al. (2007) en un mercado laboral competitivo, donde son muchas las empresas y muchos los trabajadores que demandan y ofrecen servicios de trabajo respectivamente, tiende a existir un salario único. En el caso de los trabajadores sindicalizados no existe un salario de equilibrio, como sí lo podría haber en un mercado competitivo de bienes y servicios. El sindicato constituye un monopolio de la oferta de trabajo, mientras que la empresa es un monopolio de la demanda de trabajo (o monopsonio). El salario no quedará fijado por el equilibrio entre oferta y demanda de trabajo, sino que constituirá un rango posible que dependerá de la fuerza de cada participante y de la legislación existente.
} 
La ley establece claramente ciertas condiciones para el trabajo suministrado. Algunas de estas condiciones se refieren al registro de empresas suministradoras de servicio, y a una limitación temporal de los trabajadores suministrados, la que no puede exceder de 180 días. Qué sucede entonces con las empresas suministradoras que no se han declarado como tal, es una interrogante que está aún por resolverse.

La situación concreta es que algunas empresas que se declaran contratistas en realidad no lo son. Y no lo son, porque no cumplen el requisito básico de la existencia de un vínculo laboral entre sus trabajadores contratados y éstas. La situación legal es entonces compleja, por decir lo menos, ya que se está encubriendo una relación laboral. En este caso debe realizarse una corrección de la situación para ajustarse a la ley. Y tal corrección implica que la empresa mandante debe asumir su responsabilidad legal contratando a los trabajadores subcontratistas que efectivamente trabajan bajo subordinación y dependencia de esta misma empresa mandante. Esta ha sido precisamente la situación que se ha registrado en varias oportunidades.

Consciente de la modalidad de trabajo encubierto de algunas empresas contratistas y mandantes, la Dirección del Trabajo ha reaccionado ordenando a la empresa mandante internalizar o contratar a estos trabajadores. Las empresas mandantes han apelado a la justicia para revertir esta orden, y en la mayoría de los casos se les ha dado a las empresas mandantes la razón. La Dirección del Trabajo no tendría, de acuerdo a las decisiones de los tribunales, la facultad de ordenar a las empresas mandantes la contratación de los trabajadores contratistas que tienen un vínculo laboral con la empresa mandante.

Con independencia de si la Dirección del Trabajo tiene o no tal facultad, al darse ese caso concreto, se está produciendo un encubrimiento de una relación laboral. No se está cumpliendo con el ordenamiento jurídico del país, y los trabajadores están quedando sin la adecuada protección. Ciertamente los trabajadores subcontratados por las empresas contratistas y que poseen un vínculo laboral con la empresa mandante no tienen conocimiento de los fundamentos legales y teóricos de su situación laboral. Pero sí tienen en varias ocasiones una percepción correcta de que no están siendo respetados sus derechos y de que están viviendo situaciones injustas. Se saben trabajadores de segunda categoría, y tienen la percepción de que algo no está funcionando correctamente en la legalidad. Sienten que deberían tener los mismos derechos y privilegios que sus pares contratados, sobre todo porque realizan exactamente el mismo tipo de trabajo. De continuar estas percepciones, es probable que los conflictos en las mineras se sigan repitiendo. A menos que las empresas mandantes reaccionen antes de estallar el conflicto, y ofrezcan cuantiosos bonos de remuneración a los trabajadores subcontratados. 


\section{Bibliografía}

Abramo, Lais y Marta Rangel (ed.) (2005), América Latina: negociación colectiva y equidad de género, Oficina Internacional del Trabajo, Santiago de Chile.

Acuña, Eduardo y Ernesto Pérez (2005), Trayectorias Laborales: El Tránsito entre el Trabajo Asalariado y el Empleo Independiente, Dirección del Trabajo, Santiago de Chile.

Aguilar, Omar (2003), ENCLA 2002, Relaciones de trabajo y empleo en Chile. Departamento de Estudios, Dirección del Trabajo, Santiago de Chile.

Albornoz, Marcelo (2001), "La calificación del vínculo laboral en el proyecto de reformas laborales y las distintas dimensiones de la propuesta", Boletín Dirección del Trabajo, Santiago de Chile, N 145, pp. 1-9.

Daroch, Solange (2007), Los conflictos laborales en Chile: principales ejes para la discusión, Departamento de Economía, Universidad de Chile, Santiago, manuscrito no publicado.

De la Garza, Enrique (coord.) (2006), Teorías Sociales y Estudios del Trabajo: Nuevos Enfoques, Cuadernos A, Anhropos Editorial, Barcelona.

De Laire, Fernando (1999), La trama invisible o los claroscuros de la flexibilidad, Cuadernos de Investigación número 8, Departamento de Estudios, Dirección del Trabajo, Santiago de Chile.

Dirección del Trabajo (2007), ENCLA 2006 Informe de resultados. Quinta Encuesta Laboral, Gobierno de Chile, Dirección del Trabajo, División de Estudios, Santiago de Chile.

Echeverría, Magdalena (2006), Los riesgos laborales de la subcontratación, Aporte al debate laboral número 19, Departamento de Estudios, Dirección del Trabajo, Santiago de Chile.

Ídem (1997), “Subcontratación de la producción y subcontratación del trabajo”, en Temas Laborales, Año 3, N 7, Dirección del Trabajo, Santiago de Chile.

Echeverría, Magdalena et al. (2001), (reedición), El otro trabajo. El suministro de personas en las empresas, Cuaderno de Investigación $\mathrm{N}^{\circ} 7$, Departamento de Estudios, Dirección del Trabajo, Santiago de Chile.

Echeverría, Magdalena y Verónica Uribe-Echevarría, (1998), Condiciones de Trabajo en Sistema de Subcontratación, ETM, OIT-Chile, Documento $\mathrm{N}^{\mathrm{o}}$ 81, Santiago de Chile. 
Emmerich, Thalos (1999), Atypische Beschäftigungsformen in Europa, Manz Verlag, Viena.

Feres, María Ester, Helia Henríquez y José Luis Ugarte (1999), Protección de los trabajadores. Estudio Nacional de Chile, Agosto 1999, manuscrito no publicado, en: www.ilo.org/public/english/dialogue/ifpdial/downloads/ wpnr/chile.pdf, accedido el 11.10.2004.

Gálvez, Thelma (2001), Para Reclasificar el Empleo: Lo Clásico y lo Nuevo, Cuaderno de Investigación número 14, Departamento de Estudios, Dirección del Trabajo, Santiago de Chile.

Henríquez, Helia y Verónica Riquelme (2006), Lejos del Trabajo Decente: El empleo desprotegido en Chile, Cuaderno de Investigación $\mathrm{N}^{\circ} 30$, Departamento de Estudios, Dirección del Trabajo, Santiago de Chile.

Imbusch, Peter (1997), „Neoliberalismus und Arbeitsbeziehungen in Chile - Die Erfahrungen mit der Pinochet-Diktatur“, en: Peter Imbusch et al. (editores), Neoliberalismus und Arbeitsbeziehungen in Lateinamerika, Vervuert, Frankfurt am Main.

Infante, Ricardo y Emilio Klein (1997), Exclusión social, OIT, ETM, Santiago de Chile.

International Labour Organisation (2003), Die Erstreckungsbereich des Arbeitsverhältnis, Genf, ILO, en http://www.ilo.org/wcmsp5/groups/public/ —ed_norm/— relconf/documents/meetingdocument/wcms_ilc_91_repv_de.pdf, accedido el 15.12.2007.

Iranzo, Consuelo y Jacqueline Richter (2008), "Subcontratación y conflicto. El caso de la Siderurgia del Orinoco”, en Revista Gaceta Laboral, Revista de la Universidad del Zulia, Maracaibo, Volumen 14, N 1, pp. 5-34.

Ídem (2005), La subcontratación laboral. Bomba de tiempo contra la paz social, Editorial Universidad Central de Venezuela - Centro de Estudios del Desarrollo, Caracas.

Leiva, Sandra (2006), Abhängig Selbständige: Chronik einer angekündigten Prekarität - Deutschland und Chile im Vergleich, Tesis de Doctorado en Sociología, Universidad de Göttingen.

Ídem (2004), “Exclusión social en el mercado laboral en Chile en la década de los 90", en Lateinamerika-Studien 47, Vervuert Verlag, Frankfurt am Main.

Ídem (2000), Part-time work in Chile: is it precarious employment? Women and Development Unit Series, $\mathrm{N}^{\circ} 26$ (LC/L. 1301-P), Economic Commission for Latin America and the Caribbean, Santiago, Chile. 
Leiva, Sandra, Jaime Sperberg y Dirk Koob (2002), „Exklusionsprozesse in Lateinamerika. Arbeitsmarkt-und alterssicherungsbedingte Ausgrenzung am Beispiel Chiles und Uruguays“, en Lateinamerika-Analysen, Número 3, Institut für Iberoamerika-Kunde, Hamburgo, pp. 3-28.

Ley de subcontratación 20123.

Mc Connell, Campbell et al. (2007), Economía Laboral, $7^{\mathrm{a}}$ edición, McGraw Hill, Interamericana de España.

Organización Internacional del Trabajo (1997), Trabajo en régimen de subcontratación. Informe VI (1), Conferencia Internacional del Trabajo, $85^{\mathrm{a}}$ Reunión, OIT, Ginebra.

Reinecke, Gerhard (2000), Inside the Model: Politics, Enterprise Strategies and Employment Quality in Chile, Hamburg Universität, Tesis de Doctorado.

Rodgers, Gerry (1989), "Precarious work in Western Europe: The state of de debate”, en Rodgers, Gerry and Janine Rodgers (Eds.) Precarious jobs in labour market regulation: The growth of atypical employment in Western Europe, International Labour Organisation / International Institute for Labour Studies, Geneva.

Silva, Consuelo (2007), La Subcontratación en Chile: Aproximación sectorial, Documento Preparado para el Consejo Asesor Presidencial Trabajo y Equidad, manuscrito no publicado.

Supiot, Alain (2001), Beyond Employment: Changes in Work and the Future of Labour Law in Europe. A Report prepared for the European Commission, Oxford.

Thiery, Peter (2000), Transformation in Chile: institutioneller Wandel, Entwicklung und Demokratie, 1973-1996, Vervuert, Frankfurt am Main.

Ugarte, José Luis (2008), “Inspección de Trabajo en Chile: vicisitudes y desafíos”, en Revista Latinoamericana de Derecho Social, UNAM, México, número 6, enero-junio de 2008, pp. 187-204.

Ídem (2006), "Sobre relaciones laborales triangulares: La subcontratación y el suministro de trabajadores”, en Ius et Praxis, Revista de la Universidad de Talca, Talca, $\mathrm{N}^{\circ}$ 12, Vol. 1, pp. 11-29.

Ídem (1999), “Suministro de trabajadores y trabajo temporal. ¿Empresas sin trabajadores?” en ¿Empresas sin trabajadores? Legislación sobre las empresas de trabajo temporal, pp. 16-38, Cuaderno de Investigación N ${ }^{\circ}$ 10, Dirección del Trabajo, Santiago de Chile. 
Valencia, Lucía et al. (1998), Economía, Editorial Santillana del Pacífico, Santiago de Chile.

Walker, Francisco y Ricardo Liendo (2004), “Algunas reflexiones sobre los aspectos jurídico-laborales de la Externalización (Outsourcing)”, en: Revista Economía \& Administración, Santiago de Chile, pp. 60-67. 